Central Washington University

ScholarWorks@CWU

All Faculty Scholarship for the College of the Sciences

$5-29-2012$

Resonance frequencies of a spherical aluminum shell subject to static internal pressure

Andrew A. Piacsek

Sami Abdul-Wahid

Robert Taylor

Follow this and additional works at: https://digitalcommons.cwu.edu/cotsfac

Part of the Acoustics, Dynamics, and Controls Commons 


\title{
Resonance frequencies of a spherical aluminum shell subject to static internal pressure
}

\author{
Andrew A. Piacsek and Sami Abdul-Wahid \\ Department of Physics, Central Washington University, Ellensburg, Washington 98926 \\ piacsek@cwu.edu,sipho.1989@gmail.com \\ Robert Taylor \\ Department of Industrial Engineering and Technology, Central Washington University, \\ Ellensburg, Washington 98926 \\ terebigemuwan@gmail.com
}

\begin{abstract}
Measurements of the vibrational response of a spherical aluminum shell subject to changes in the interior pressure clearly demonstrate that resonance frequencies shift higher as the pressure is increased. The frequency shift appears to be smaller for longitudinal modes than for bending wave modes. The magnitude of frequency shift is comparable to analytical predictions made for thin cylindrical shells. Changes in the amplitudes of resonance peaks are also observed. A possible application of this result is a method for noninvasively monitoring pressure changes inside sealed containers, including intracranial pressure in humans.

(C) 2012 Acoustical Society of America

PACS numbers: 43.40.Ey, 43.25.Gf [JGM]

Date Received: February 16, 2012 Date Accepted: April 20, 2012
\end{abstract}

\section{Introduction}

Recent interest in developing noninvasive techniques for measuring intracranial pressure (ICP) has led to investigations of the vibrational response of skulls subject to internal pressure changes. A significant result of these limited studies, both experimental ${ }^{1}$ and computational, ${ }^{2}$ is that an increase in ICP produces an upward shift in certain resonance frequencies of ovine and human skulls. Although the maximum ICP investigated was less than $0.5 \mathrm{psi}$, this result is consistent with the predictions of early theoretical studies of thin cylindrical shells subject to much larger pressures. ${ }^{3-10}$ In addition to ICP monitoring, this phenomenon has the potential to be exploited as a diagnostic tool for the pressure inside any thin-walled container that should remain sealed, such as an industrial tank containing hazardous waste.

It should be emphasized that we are concerned only with the effect on shell resonance frequencies due to static internal pressure, regardless of the specific fluid contained in the shell, rather than acoustic pressures associated with spherical cavity modes. The mechanism by which prestress within a shell causes the resonance frequencies to shift is clearly nonlinear. In shell vibrations, there are two distinct sources of nonlinearity: the interaction of shell curvature and finite thickness gives rise to nonlinear straindisplacement relations ${ }^{5}$ ("geometric nonlinearity"), and the stress-strain relations may be nonlinear ("physical nonlinearity"). ${ }^{11,12}$ Both effects can be simultaneously incorporated into a three-dimensional nonlinear theory of elasticity, as described in Refs. 4 and 9. In doing so, however, an intuitive understanding of the role of nonlinearity is obscured and the results can be interpreted only in the context of the specific geometry for which the equations of motion were derived. For example, the theoretical predictions of Refs. 5 and 9 and the experimental results of Refs. 3 and 6 show that flexural modes are more strongly affected by internal pressure than longitudinal modes, the size of the frequency shift for a given mode depends on the cylinder radius to length ratio.

Although cylinders are an appropriate model for many structural applications, expanding the study to other shell shapes, and measuring response changes over a 
wide range of pressures, may improve our physical insight into the nonlinear mechanism connecting prestress to shell response. To our knowledge, the resonance behavior of spherical shells subject to prestress has not been investigated, either theoretically or experimentally.

This letter describes a simple experiment that clearly demonstrates changes in the vibrational response of a uniform spherical aluminum shell that is subject to a wide range of internal pressures. The increase in resonance frequency, as well as changes in peak amplitudes, with internal pressure is measured for both an air-filled and a water-filled shell. Detailed observations are provided of the effect on resonance behavior of the change in pressure. The measured frequency shifts are compared with analytical predictions of cylindrical shells subject to internal pressure.

\section{Experiment}

The experimental apparatus for measuring the resonance frequencies of a spherical shell is shown in Fig. 1. The test body was a uniform spherical shell of radius 3.0 in. $(7.62 \mathrm{~cm})$ and thickness $1 / 8$ in. $(0.3175 \mathrm{~cm})$ made of aluminum (ASTM B209), with a threaded $3 / 8$ in. hole. The shell was suspended with elastic string tied to a pair of hooks inserted into the hole. When supporting the weight of the empty shell, the string has a fundamental frequency that is three orders of magnitude smaller than the shell frequencies of interest, indicating that coupling between shell vibrations and the suspension system should be weak.

An acoustic driver with a horn (Peavey, model CH6-9) mounted underneath the shell provided the excitation and two small accelerometers, one uniaxial (Dytran model 3032A) and one triaxial (Dytran model 3053B), mounted on the shell provided a means for measuring the vibration response. The acoustic excitation was chosen to avoid the mass loading effects of a mechanical shaker; the location of the driver was intended to excite shell modes that are axisymmetric with respect to the gravity vector.

The acoustic driver received a swept sinusoidal signal from a National Instruments PXI-5402 function generator, amplified by a Labworks model PA-119 linear amplifier. The accelerometer signals were conditioned by a National Instruments PXI4472B data acquisition system. A microphone mounted between the driver and shell provided a way to monitor the amplitude of the driving signal. The function generator output and the input signals were managed by a LabView virtual instrument.

To maintain pressure inside the shell, a valve was threaded into the $1 / 8 \mathrm{in}$. hole in the shell. With water inside the shell, a valve that accommodates a hydraulic

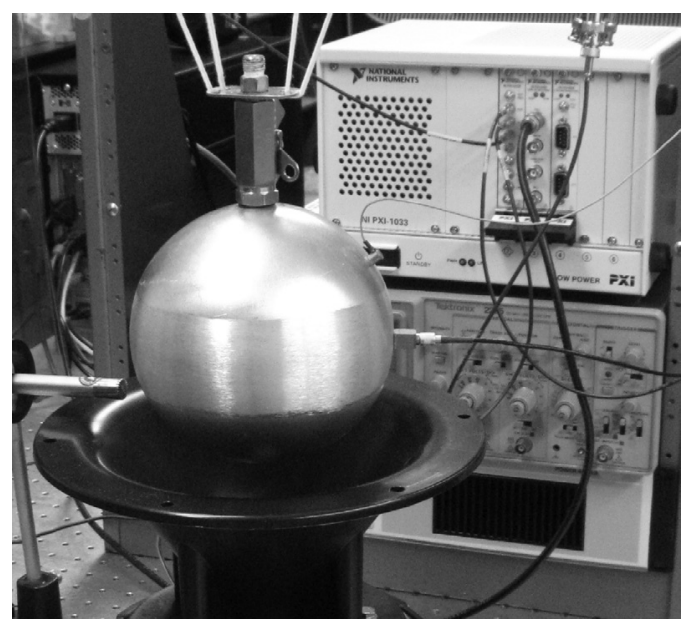

Fig. 1. Photograph of apparatus to measure the resonance frequencies of a pressurized spherical shell. A pressure valve is threaded into the shell at the top; connected to this is the elastic string suspension system. 
hand pump (Ralston, model HPGV with gauge) was used. With air in the shell, a valve that accommodates plastic tubing connected to a bicycle pump was used.

The frequency response of the water-filled shell with attached valve was measured between $7.0-22.0 \mathrm{kHz}$ with the water at atmospheric pressure. From this spectrum, two distinct sets of peaks near 16.4 and $18 \mathrm{kHz}$ were chosen for further investigation with increased interior pressure. The frequency response of the air-filled shell (with valve) was measured between 15 and $18 \mathrm{kHz}$, leading to the identification of a strong peak near $16 \mathrm{kHz}$ used in the changing pressure measurements.

For the two sets of peaks in the water-filled shell spectrum, the frequency response was obtained using a step size of $1 \mathrm{~Hz}$ with internal pressures of 20 to 160 psig in increments of 20 psig. For context, these pressures are comparable to those found in bicycle tires. The water pump gauge is calibrated in increments of $5 \mathrm{psi}$; the uncertainty for the actual pressure inside the shell is about $+/-2$ psi. The response of the air-filled shell also had a step size of $1 \mathrm{~Hz}$, but mechanical issues with the pump and hose system limited reliable measurements to pressures between 20 and 60 psi. Both types of pumps also allowed for the pressure to be decreased in a controlled way.

With either pump remaining connected to the shell throughout the measurement and the valve left open, only a gentle squeeze of the pump handle was required between measurements, leaving the shell undisturbed. This was important to do, since preliminary measurements showed that the shell response is very sensitive to changes in the suspension system, contrary to our initial expectations. Lifting or pushing down on the shell, as might occur when the valve was being opened or closed, could produce significant changes in the amplitude of peaks in the response curve. We also observed that the response curve of the water-filled shell could evolve over a period of hours, which we attribute to the elastic string stretching out and becoming stiffer. The sensitivity of the shell's vibrations response to fluctuations in the suspension (or in the shell's weight) is illustrated by oscillations that appear in the spectrum if the shell is slightly swinging during data collection.

To confirm that observed changes in the shell response were due only to pressure changes, and not due to gradual changes in the suspension or other environmental effects, the response was measured after each 20 psi increase in the pressure, then again as the pressure was decreased.

\section{Results}

The response of the water-filled shell near $16.4 \mathrm{kHz}$ at each of the several pressure values tested is shown in Fig. 2. The pressure values shown are gauge pressure, which is the pressure above atmospheric pressure. Three developments are clearly seen in this plot as the internal pressure is increased: every peak is shifted to higher resonance frequencies; there is a consistent change in the amplitude of each peak; and some peaks split or merge.

At the lowest pressure (20 psig), there are three distinct peaks, with a fourth that is less distinct, within the $200 \mathrm{~Hz}$ range plotted. The first peak, at $16368 \mathrm{~Hz}$, is relatively narrow and tall and is followed by a sharp antiresonance. The second peak, at $16399 \mathrm{~Hz}$, has a noticeable shoulder; the third distinct peak is at $16414 \mathrm{~Hz}$. A fourth peak may be discerned at $16462 \mathrm{~Hz}$. A sharp antiresonance occurs at $16383 \mathrm{~Hz}$. Multiple peaks associated with a particular shell mode likely result from asymmetries due to the presence of the valve and the suspension system.

As the interior pressure is increased, all four peaks shift to the right (higher frequencies). The first peak, however, decreases in amplitude and eventually splits into two peaks, while the second peak increases in amplitude and loses the shoulder. The third peak diminishes in amplitude and eventually becomes absorbed into the second peak; the fourth peak increases in amplitude. The peak frequencies vs. internal pressure of the four resonances described in Fig. 2 are listed in Table 1.

Another set of peaks near $18 \mathrm{kHz}$ exhibits similar behavior, as shown in Fig. 3. The amplitude of the peak near $18340 \mathrm{~Hz}$ grows significantly with pressure, while 


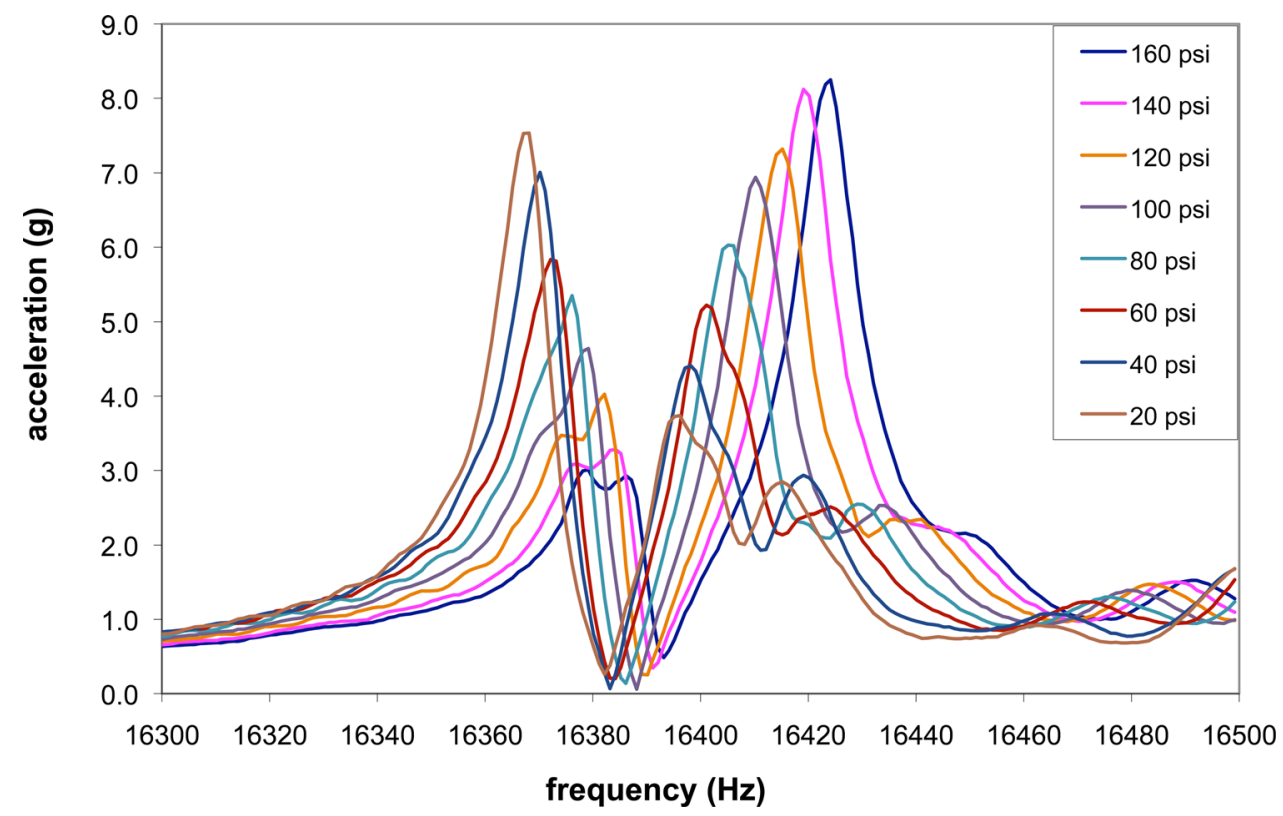

Fig. 2. (Color online) Frequency response near $16 \mathrm{kHz}$ of an aluminum spherical shell filled with water at several values of interior gauge pressure. Peaks shift to higher frequencies and change amplitude as pressure increases. The legend indicates gauge pressure.

the peak near $18100 \mathrm{~Hz}$ is relatively unchanged. Also of interest is the development of a sharp antiresonance near $18300 \mathrm{~Hz}$ between 50 and 100 psig. The early portion of the response curves in Figs. 2 and 3 exhibit the oscillations associated with a slightly swinging apparatus.

The air-filled shell resonance near $16 \mathrm{kHz}$ was measured with internal pressures at 20, 40, and 60 psig. The response curve contains two significant peaks: over the 40 psi range tested, the taller peak shifted from 16030 to $16040 \mathrm{~Hz}$ while remaining nearly constant in amplitude; the much smaller peak grew eight-fold in amplitude while remaining essentially unchanged in frequency.

\section{Discussion}

Measurements of both the air-filled and water-filled shell show that resonance frequencies increase as the internal pressure is increased. Over the range of pressures tested (20-160 psi), the relationship was approximately linear for several resonance peaks between 16 and $18 \mathrm{kHz}$. This result is consistent with the predictions of Refs. 5 and 8

Table 1. Observed resonance frequencies in a spherical aluminum shell filled with water at different internal pressures.

\begin{tabular}{lcccc}
\hline \hline Pressure (psig) & First peak & Second peak & Third peak & Fourth peak \\
\hline 20 & 16368 & 16396 & 16415 & 16462 \\
40 & 16370 & 16398 & 16419 & 16465 \\
60 & 16372 & 16401 & 16424 & 16471 \\
80 & 16376 & 16405 & 16429 & 16475 \\
100 & 16379 & 16410 & 16433 & 16479 \\
120 & 16382 & 16415 & 16436 & 16483 \\
140 & 16384 & 16419 & 16437 & 16488 \\
160 & 16386 & 16424 & 16449 & 16491 \\
\hline
\end{tabular}




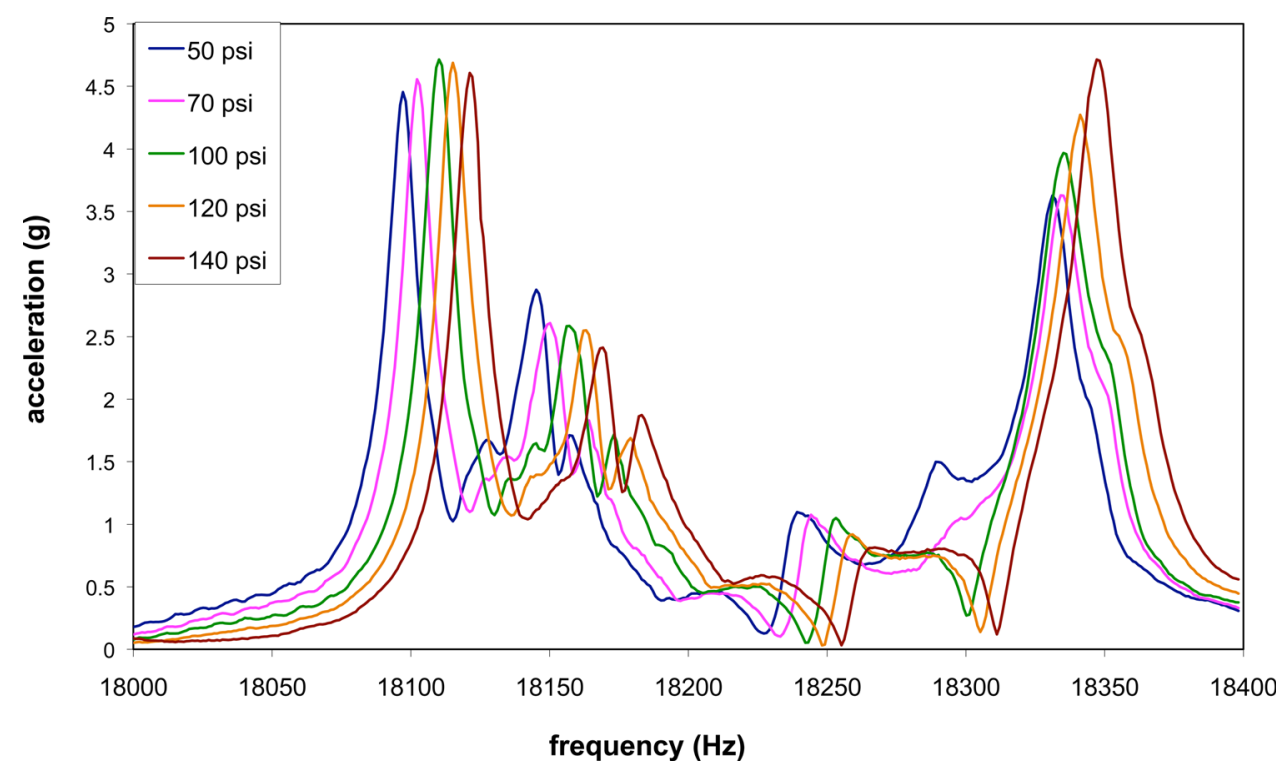

Fig. 3. (Color online) Frequency response near $18 \mathrm{kHz}$ of an aluminum spherical shell filled with water at several values of interior gauge pressure, showing the evolution of four distinct resonance peaks. The legend indicates gauge pressure.

for cylindrical shells subject to small pressures. The average slope of frequency shift vs pressure $\left(\Delta f_{\mathrm{pk}} / \Delta p\right)$ for these six peaks is shown in Table 2 . The shift in the $16.37 \mathrm{kHz}$ peak is only half that of the $18.1 \mathrm{kHz}$ peaks, which might be explained by differences in the vibrational mode types. Results of a finite- element model of the aluminum shell filled with water, as well as eigenfrequency calculations for a fluid-filled spherical shell undergoing axisymmetric vibrations, ${ }^{13,14}$ suggest that the peaks near $18 \mathrm{kHz}$ are possibly degenerate versions of the $n=12$ lowest branch mode (primarily bending waves), while the peaks near $16.37 \mathrm{kHz}$ may comprise the $n=2$ mode from the next higher branch (primarily longitudinal waves). Studies on cylindrical shells also report that internal pressure affects transverse modes more strongly than longitudinal modes., ${ }^{3,5,6,9}$

The magnitude of the observed frequency shifts in the spherical shell can be indirectly compared with the analytical predictions of Refs. 5 and 7 which are applicable to cylindrical shells. With $\omega_{0}$ representing the angular frequency at zero internal gauge pressure, the predicted frequency due to internal pressure $p$ can be written $\omega=\sqrt{\omega_{0}^{2}+\Delta \omega^{2}}$, where

$$
\Delta \omega^{2}=\frac{n^{2} p}{\rho h R}
$$

for purely circumferential waves (axial wavenumber $m=0$ ). Here, $\rho, h$, and $R$ are the density, thickness, and radius, respectively, of the spherical or cylindrical shell. In Ref. $5, n^{2}$ is replaced by $\left(n^{2}-1\right)^{2} /\left(n^{2}+1\right)$; these expressions differ by more than $5 \%$ only for

Table 2. Measured resonance frequency shift vs change in internal pressure for several different resonance peaks. Units are $\mathrm{Hz} / \mathrm{psi}$.

\begin{tabular}{ccccccc}
\hline \hline Peak $(\mathrm{Hz})$ & Water 16370 & Water 16410 & Water 18100 & Water 18150 & Water 18350 & Air 16000 \\
\hline$\Delta f_{\mathrm{pk}} / \Delta p$ & 0.13 & 0.2 & 0.27 & 0.27 & 0.18 & 0.26 \\
\hline \hline
\end{tabular}


$n<10$. When applied to the spherical aluminum shell, Eq. (1) gives good agreement with the observed frequency shifts if a suitable value of $n$ is chosen. For example, the measured peaks near $18.1 \mathrm{kHz}$ (shown in Fig. 3), which are close to the predicted $n=12$ mode, are shifted by $24 \mathrm{~Hz}$ as the internal pressure is increased by $90 \mathrm{psi}$, whereas Eq. (1) predicts a shift of $25 \mathrm{~Hz}$ if $n=6$. Similarly, the shift seen in the $16.4 \mathrm{kHz}$ peak $(n=11)$ is matched by Eq. (1) if $n$ is set to 5 . For the air-filled shell, the observed shift from 16025 to $16035 \mathrm{~Hz}$ is obtained theoretically if $n=6$, although this resonance corresponds to the $n=9$ mode for an empty shell.

The significance of these comparisons is that the spherical shell exhibits a frequency shift that is approximately linear with the increase in internal pressure, consistent with cylindrical shell theory despite the difference in geometry. Our results also confirm the prediction expressed in Eq. (1) that the frequency shift due to internal pressure changes is not directly related to the composition of the internal fluid. Larger fluid densities will result in lower frequencies for a given flexural mode of vibration at zero internal pressure, but the frequency shift that occurs when the pressure is increased is independent of fluid properties.

It should be noted that in Refs. 3-6 an internal pressure change of 140 psi was at the low end of pressures measured or used in the calculations, whereas the overall range of ICP is on the order of 1 psi. The current study involves an intermediate range of pressures. Curiously, the study on ovine skulls also reported a linear relationship between ICP and changes in the impedance spectrum, but only up to a pressure of $15 \mathrm{~mm} \mathrm{Hg}\left(0.29\right.$ psi); no changes were observed at higher pressures. ${ }^{1}$

In addition to frequency shifts, the amplitudes of resonance peaks also exhibited a regular change with increased or decreased internal pressure. Much care had to be taken not to disturb the shell and its suspension system during the course of the experiment, since even a small displacement could affect both the frequency and amplitude of resonance peaks.

It is important to note that the weight of the water inside the shell contributed a nonuniform stress within the shell that was comparable to the internal pressure changes. The magnitude of the stress at the shell's equator due to the water's weight is $45 \mathrm{psi}$, which is equivalent to the extra tensile stress produced by an internal pressure of about 4 psi.

\section{Conclusion}

Experiments with a spherical aluminum shell filled with either air or water clearly demonstrate that internal fluid pressure has a measurable effect on the vibration response of the shell. On average, shell resonance frequencies, as measured by accelerometers, shifted higher by about $2 \mathrm{~Hz}$ as the internal pressure was increased by 10 psi over a range of 140 psi, although this rate varied by as much as a factor of 2 for different resonance peaks. Peak amplitudes were also observed to change, in a and reversible way, with internal pressure.

The observed frequency shifts appear to be consistent with the early analysis of thin cylindrical shells based on nonlinear geometrical theory. However, due to the fundamental role played by the shell geometry in its nonlinear behavior, comparison between spherical and cylindrical shells is not exact. This fact suggests that a generalized approach to predicting the effect of internal pressure on resonance frequencies for any shell shape is not possible. Therefore, applying a finite-element model for each shell geometry of interest may be the best approach for accurately predicting frequency shifts.

Resonance frequency shifts may provide a mechanism for noninvasively monitoring ICP changes in patients at risk for intracranial hypertension. Among the several challenges facing implementation of this approach are the sensitivity of the vibration response to factors such as the weight distribution and interaction with the shell support, the variation of cranial morphologies among patients, and the ability to detect very small frequency shifts. Finite element models that incorporate data from a 
patient's CT or MRI scan may provide useful guidance in determining when changes in ICP have reached a critical level.

\section{Acknowledgments}

Financial support was provided by the National Science Foundation Science Talent Expansion Program (Award No. 0653094), the Central Washington University Science Honors Research Program, and the Washington Space Grant Consortium Award No. NNX10AK64H. Dr. P. Mark Li initially posed the interesting question that led to this research.

\section{References and links}

${ }^{1}$ M. Stevanovic, G. Wodicka, J. Bourland, G. Graber, K. Foster, G. Lantz, W. Tacker, and

A. Cymerman, "The effect of elevated intracranial pressure on the vibrational response of the ovine head," Ann. Biomed. Eng. 23, 720-727 (1995).

${ }^{2} \mathrm{Z}$. Li and Y. Luo, "Finite element study of correlation between intracranial pressure and external vibration responses of human head," Adv. Theor. Appl. Mech. 3, 139-149 (2010).

${ }^{3}$ Y. C. Fung, E. E. Sechler, and A. Kaplan, "On the vibration of thin cylindrical shells under internal pressure," J. Aeronaut. Sci. 24, 650-660 (1957).

${ }^{4}$ G. Herrmann and A. E. Armenakas, "Dynamic behavior of cylindrical shells under initial stress," Report No. AFOSR TN-60-425, Air Force Office of Scientific Research (1960).

${ }^{5}$ A. E. Armenakas, "Influence of initial stress on the vibrations of simply supported circular cylindrical shells," AIAA J. 2, 1607-1612 (1964).

${ }^{6}$ R. Miserentino and L. F. Vosteen, "Vibration tests of pressurized thin-walled cylindrical shells," Technical Note TN D-3066, NASA (1965).

${ }^{7} \mathrm{~J}$. Greenspon, "Vibrations of thick and think cylindrical shells surrounded by water," J. Acoust. Soc. Am. 10, 1321-1328 (1961).

${ }^{8} \mathrm{~J}$. Greenspon, "Effect of external or internal static pressure on the natural frequencies of unstiffened, cross-stiffened, and sandwich cylindrical shells," J. Acoust. Soc. Am. 39, 407-408 (1965).

${ }^{9}$ P. R. DiGiovanni and J. Dugundji, "Vibrations of freely supported orthotropic cylindrical shells under internal pressure,” Report No. 65-0640, Air Force Office of Scientific Research (1965).

${ }^{10}$ R. F. Keltie, "The effect of hydrostatic pressure fields on the structural and acoustic response of cylindrical shells," J. Acoust. Soc. Am. 79, 595-603 (1986).

${ }^{11}$ H. Kraus, Thin Elastic Shells (Wiley, New York, 1967).

${ }^{12}$ K. M. Mushtari and K. Z. Galimov, Nonlinear Theory of Thin Elastic Shells (Tatknigoizdat, Kazan, 1957).

${ }^{13}$ M. C. Junger and D. Feit, Sound, Structures, and Their Interaction, 2nd ed. (MIT Press, Cambridge, 1986), p. 230.

${ }^{14}$ A. E. Engin and Y. K. Liu, “Axisymmetric response of a fluid-filled spherical shell in free vibrations," J. Biomechanics 3, 11-22 (1970). 